\title{
A TAUBERIAN THEOREM AND ITS PROBABILITY INTERPRETATION
}

\author{
BY \\ FRANK SPITZER(')
}

Introduction. The purpose of this paper is to extend part of the theory of identically distributed independent random variables, continuing the work in [9] and [10]. [9] was devoted to the solution of a problem of immediate probabilistic interest: if $X_{i}$ are identically distributed independent random variables, with partial sums

$$
S_{0}=0, \quad S_{n}=X_{1}+\cdots+X_{n}, \quad n \geqq 1,
$$

and

$$
M_{n}=\max _{0 \leqq \boldsymbol{k} \leqq n} S_{k},
$$

it was possible, by simple methods, to find the generating functions of the distributions of the random variables $M_{n}$. In [10] it was shown that these probabilistic results are closely related to certain problems in analysis; they yield information concerning certain solutions of the Wiener-Hopf equation

$$
F(x)=\int_{0}^{\infty} k(x-y) F(y) d y,
$$

where $k(x)$ is the (known) density of the random variables $X_{i}$.

We begin by summarizing some central results of [10], in order to then explain in what fashion they are extended in this paper.

TheOREM. Let $k(x)$ be a symmetric probability density, with finite variance $\sigma^{2}$, i.e.

$$
k(x)=k(-x) \geqq 0, \quad \int_{-\infty}^{\infty} k(x) d x=1, \quad \int_{-\infty}^{\infty} x^{2} k(x) d x=\sigma^{2} .
$$

Suppose a solution of $(0.1)$ is defined to mean a nondecreasing function $F(x)$, defined on $0 \leqq x<\infty$, continuous on the right, with $F(0)>0$. Then equation (0.1) has (except for a multiplicative constant) a unique solution $F(x)$. The iterates of (0.1), defined by

Presented to the Society, November 29, 1958 under the title An extension of the renewal theorem; received by the editors, January 2, 1959.

(1) Research supported by the ONR at the University of Minnesota. 


$$
\begin{aligned}
F_{0}(x) & =1, & x \geqq 0, \\
F_{n+1}(x) & =\int_{0}^{\infty} k(x-y) F_{n}(y) d y, & x \geqq 0,
\end{aligned}
$$

converge to a solution $G(x)$ in the following way:

$$
G(x)=\lim _{n \rightarrow \infty}(n \pi)^{1 / 2} F_{n}(x)
$$

satisfies (0.1), is absolutely continuous, nondecreasing, with $G(0)>0$.

This solution $G(x)$ has the asymptotic behavior

$$
\lim _{x \rightarrow \infty} \frac{G(x)}{x}=\frac{2^{1 / 2}}{\sigma} .
$$

Now it is possible to formulate the central result of this paper as

THEOREM 1. The above theorem remains valid in every respect if

(a) the hypothesis that $k(x)=k(-x)$ is weakened to

$$
\int_{-\infty}^{\infty} x k(x) d x=0
$$

and

(b) the conclusion that $\lim _{x \rightarrow \infty} G(x) / x=2^{1 / 2} / \sigma$ is strengthened to

$$
\lim _{x \rightarrow \infty}[G(x+h)-G(x)]=h \frac{2^{1 / 2}}{\sigma} .
$$

The methods of this paper are those of [10] with obvious extensions, and with one important exception. In [10], where $k(x)=k(-x)$ it was possible to show that the solution $G(x)$ of $(0.1)$ has the Laplace transform

$$
\int_{0-}^{\infty} e^{-\lambda x} d G(x)=\exp \left\{-\frac{1}{2 \pi} \int_{-\infty}^{\infty} \frac{\lambda}{\lambda^{2}+u^{2}} \log [1-\phi(u)] d u\right\},
$$

where $\phi(u)$ is the characteristic function of the random variables $X_{i}$, i.e.

$$
\phi(u)=\int_{-\infty}^{\infty} e^{i u x} k(x) d x,
$$

and the left hand side of $(0.4)$ is understood to mean

$$
\int_{0-}^{\infty} e^{-\lambda x} d G(x)=G(0)+\int_{0+}^{\infty} e^{-\lambda x} d G(x) .
$$

In [10], Karamata's Tauberian theorem applied to (0.4) easily yielded the asymptotic result $(0.2)$. 
In the present case, when $k(x)$ is not even, so that $\phi(u)$ is not real valued, it is not possible to obtain such an elegant analytic representation for $G(x)$ as equation (0.4). But one can obtain a representation which is probabilistically interesting, namely

$$
\int_{0-}^{\infty} e^{-\lambda x} d G(x)=c \cdot \exp \left\{\sum_{1}^{\infty} \frac{1}{k} E\left[e^{-\lambda S_{k}} ; S_{k}>0\right]\right\}=\frac{c}{1-E\left[e^{-\lambda Z}\right]} .
$$

Here we have to explain the positive constant $c$, depending on $k(x)$, and defined by

$$
c=\exp \left\{\sum_{1}^{\infty} \frac{1}{k}\left[\frac{1}{2}-\operatorname{Pr}\left(S_{k}>0\right)\right]\right\},
$$

and some streamlined notation: If $d P(\omega)$ is the probability measure on the infinite product space whose coordinates are the random variables $X_{i}(\omega)$ $=\omega_{i}$, then

$$
E\left[\exp \left\{-\lambda S_{k}\right\} ; S_{k}>0\right]=\int_{\left[\omega \mid \Sigma_{1}{ }^{k} \omega_{i}>0\right]} \exp \left\{-\lambda \sum_{1}^{k} \omega_{i}\right\} d P(\omega) .
$$

In the last term of $(0.5), Z$ is the following random variable:

$$
Z(\omega)=\omega_{1}+\cdots+\omega_{k}=S_{k} \text { if } S_{1} \leqq 0, \cdots, S_{k-1} \leqq 0, S_{k}>0,
$$

i.e. simply the first positive partial sum. By well known results on recurrence $Z(\omega)$ is finite with probability one when $E\left(x_{i}\right) \geqq 0$.

This random variable was first studied by Baxter [2], who obtained the equality of the second and third terms in (0.5). Our interest in the matter will be that it is possible to show an apparently new result, that

$$
E(Z)=\frac{c \sigma}{2^{1 / 2}} .
$$

Using (0.5) and (0.6), the proof of $(0.3)$ is easily accomplished by applying the renewal theorem.

The proof of Theorem 1, and in passing, of equations (0.5), (0.6), and some other new and probabilistically interesting results will be given in the first five sections. In particular we mention a strengthening of the arc-sine law in $\S 3$, and some results in $\$ 6$ which are tantamount to an extension of renewal theory in the following way: If $X_{i}$ are identically distributed nonlattice random variables with mean $\mu>0$, and $S_{n}$ their partial sums, the renewal theorem [3] states that

$$
\lim _{x \rightarrow \infty} \sum_{n=0}^{\infty} \operatorname{Pr}\left[x<S_{n} \leqq x+h\right]=\frac{h}{\mu} .
$$


It will be shown that, still for $\mu>0$, if $M_{n}=\max _{0 \leq k \leq n} S_{k}$,

$$
\lim _{x \rightarrow \infty} \sum_{n=0}^{\infty} \operatorname{Pr}\left[x<M_{n} \leqq x+h\right]=\frac{h}{\mu}
$$

$$
\begin{aligned}
\lim _{x \rightarrow \infty} \sum_{n=0}^{\infty} \operatorname{Pr}\left[x<S_{n} \leqq x+h ; M_{n-1}\right. & \left.<S_{n}\right] \\
& =\frac{h}{\mu} \exp \left\{-\sum_{1}^{\infty} \frac{1}{k} \operatorname{Pr}\left[S_{k} \leqq 0\right]\right\},
\end{aligned}
$$

and finally, for $\mu=0$,

$$
\lim _{x \rightarrow \infty} \sum_{n=0}^{\infty} \operatorname{Pr}\left[x<S_{n} \leqq x+h ; M_{n-1}<S_{n}\right]=\frac{2^{1 / 2} h}{c \sigma} .
$$

Actually matters are slightly more complicated than we have admitted so far. Equation (0.1) only makes sense for random variables with densities, and we shall find it natural to study instead equation (1.1), thus obtaining even Theorem 1 as a special case of results concerning equation (1.1). These results are contained in Theorems $5.1 \mathrm{a}$ and $5.2 \mathrm{~A}$ for nonlattice random variables, and in Theorem $5.1 \mathrm{~b}$ and $5.2 \mathrm{~B}$ which are valid for lattice random variables.

A future publication in collaboration with C. Stone, devoted to the theory of Toeplitz matrices and the corresponding lattice random variables [11], will begin where the present theory leaves off, i.e. with Theorem 5.2B.

1. We begin by discussing the equation

$$
F(x)=\int_{0}^{\infty} K(x-y) d F(y), \quad x \geqq 0 .
$$

The equation will be meaningful under the following assumptions, adhered to from now on, which will make the integral on the right well defined even as a Riemann Stieltjes' integral.

(a) the (known) function $K(x)$ is nondecreasing and continuous on the right, for $-\infty<x<\infty$,

(b) the (unknown) solution $F(x)$, if one exists, is to be nondecreasing, and also continuous on the right for $x \geqq 0$. by

(c) The contribution at $y=0$ to the integral in (1.1) is defined explicitly

$$
\int_{0}^{\infty} K(x-y) d F(y)=K(x) F(0)+\int_{0+}^{\infty} K(x-y) d F(y) .
$$

Finally we introduce the assumption that $K(x)$ is a probability distribution with mean zero and variance $\sigma^{2}$. 
(d)

$$
\begin{aligned}
K(-\infty) & =0, & K(\infty) & =1, \quad \int_{-\infty}^{\infty}|x| d K(x)<\infty, \\
\int_{-\infty}^{\infty} x d K(x) & =0, & 0 & <\int_{-\infty}^{\infty} x^{2} d K(x)=\sigma^{2}<\infty .
\end{aligned}
$$

Now one can show, using only the elementary theory of the RiemannStieltjes' integral, that equation (1.1) is a generalization of equation (0.1) in the following sense.

THEOREM 1.1. If $K(x)$ has a derivative (probability density) $k(x)$ then every solution $F(x)$ of (1.1) is a solution of (0.1) and every solution $F(x)$ of $(0.1)$ is a solution of (1.1).

Proof. Suppose $F(x)$ is a solution of $(0.1)$. Hence (by definition) it is nondecreasing and continuous on the right. We have

$$
\lim _{t \rightarrow \infty} \int_{t}^{\infty} k(x-y) F(y) d y=0,
$$

and, since $F(x)$ is nondecreasing,

$$
\lim _{t \rightarrow \infty} F(t) K(x-t)=0 .
$$

But then

$$
\begin{aligned}
\int_{0}^{\infty} K(x-y) d F(y)= & K(x) F(0)+\lim _{t \rightarrow \infty}[K(x-t) F(t)-K(x) F(0)] \\
& +\int_{0}^{\infty} k(x-y) F(y) d y \\
= & \int_{0}^{\infty} k(x-y) F(y) d y .
\end{aligned}
$$

Conversely, if $F(x)$ is a solution of (1.1), then the first integral in (1.2) exists and is finite. This implies that

$$
\lim _{t \rightarrow \infty} K(x-t) F(t)=L(x)<\infty,
$$

and also that the integral

$$
\int_{0}^{\infty} k(x-y) F(y) d y<\infty .
$$

But we have shown that $L(x) \equiv 0$ if the last integral exists. This proves the theorem.

In $\$ 2$ we shall give a probabilistic interpretation of equation (1.1) which will make it appear quite natural and which will be indispensable for the proof of existence and uniqueness of a solution. 
2. Let $X_{i}$ be independent identically distributed random variables with mean zero and variance $\sigma^{2}$, and let $K(x)$ denote their common distribution function. We shall further define

$$
\begin{aligned}
S_{0}=0, \quad S_{n} & =X_{1}+\cdots+X_{n}, \quad n \geqq 1, \\
M_{n} & =\max _{0 \leqq k \leqq n} S_{k} .
\end{aligned}
$$

The random variables $S_{n}$ and $M_{n}$ will occur throughout this paper, but at present we have to define another important stochastic process, already studied in detail in [10], and we shall summarize the principal results.

We define the Markov process $T_{n}$ as follows:

$$
T_{0}=0, \quad T_{n+1}=\left(T_{n}+X_{n+1}\right)^{+} \quad \text { for } n \geqq 0 .
$$

The notation is explained by remarking that $x^{+}=x$ if $x>0$ and $x^{+}=0$ if $x<0$. It is a well known fact, due to Pollaczek [8], that the random variables

$$
T_{n} \text { have the same distribution as } M_{n} \text {. }
$$

A short proof is given in [10]. Our principal result, also discussed in [10] goes somewhat deeper. It states that equation (1.1) is the invariant-measure equation of the Markov process $T_{n}$.

In picturesque language, if we think of $T_{n}$ as the position of a particle on the real line at time $n$, the transition law expressed by (2.3) becomes very simple. From a point $x \geqq 0$ the particle simply makes a transition to the point $\left(x+X_{n}\right)^{+}$, where $X_{n}$ of course is independent of the past. Thus the process is almost the same as that executed by the sequence $S_{n}$, with the important difference that the origin acts as a reflecting barrier on the left.

An invariant measure for this process is a countably additive $\sigma$-finite measure $\mu(E)$ defined on the Borel subsets of $[0, \infty)$, such that

$$
\mu(E)=\int_{0-}^{\infty} d \mu(y) \operatorname{Pr}[(X+y)+\in E] .
$$

It is clear that for the particular choice of $E=[0, x]$, with

$$
F(x)=\mu(E),
$$

equation (2.5) reduces to equation (1.1), and that the conditions (a), (b), (c), (d) are automatically fulfilled if, as we shall assume,

$$
K(x)=\operatorname{Pr}\left[X_{i} \leqq x\right], \quad E\left(X_{i}\right)=0, \quad 0<\sigma^{2}\left(X_{i}\right)=\sigma^{2} \leqq \infty .
$$

Now we come to the main result of this section.

THEOREM 2.1. The stochastic process $T_{n}$ has a unique invariant measure, and therefore equation (1.1) has a unique solution $F(x)$, if a solution is understood to be nondecreasing and continuous on the right, with $F(0)>0$. 
Proof. For the details we refer to [10]. It is shown there that the uniqueness of the invariant measure follows from a theorem of Harris in [7]. The crucial condition which makes the theorem of Harris applicable is that $\operatorname{Pr}\left[T_{n}=0\right.$ infinitely of ten $\left.\mid T_{0}=x\right]=1$, for all $x \geqq 0$. That this is true follows from the fact that $E\left(X_{i}\right)=0[9]$. The theorem of Harris together with the condition that

$$
\operatorname{Pr}\left[T_{n}=0 \text { infinitely often } \mid T_{0}=x\right]=1, x \geqq 0,
$$

also implies that the set consisting only of the origin has positive invariant measure. But the invariant measure determines $F(x)$ and in particular $F(0)$ which must be positive. Conversely $F(x)$ determines the invariant measure so that $F(x)$ must be unique (modulo a multiplicative constant) because $\mu$ is unique.

In the next section we shall obtain the results mentioned in the introduction concerning the random variable $Z$.

3. Let $Z$ be the first positive term among the partial sums $0=S_{0}, S_{1}$, $S_{2}, \cdots$, and $N$ the time at which it is assumed, i.e. $N=k$ if and only if $Z=S_{k}$. It follows from well known results on recurrence, for example in [9], that $Z$ and $N$ are random variables (finite with probability one) when, as we are assuming, $E\left(X_{i}\right) \geqq 0$.

By a fairly simple method, which I learned from D. Ray, we shall obtain the following representation, first discovered by Baxter, for the joint characteristic function of $Z$ and $N$.

Theorem 3.1 (BAXter). For $0 \leqq x<1, \lambda>0, E\left(X_{i}\right) \geqq 0$,

$$
E\left[x^{N} e^{-\lambda Z}\right]=1-\exp \left\{-\sum_{1}^{\infty} \frac{x^{k}}{k} E\left[e^{-\lambda S_{k}} ; S_{k}>0\right]\right\} .
$$

Proof. For real values of $\gamma$,

$$
\begin{aligned}
E\left[x^{N} e^{i \gamma} Z\right]= & \sum_{0}^{\infty} x^{n+1} E\left[\exp \left\{i \gamma\left(S_{n}+X_{n+1}\right)\right\} ; M_{n}=0 ; S_{n}+X_{n+1}>0\right] \\
= & \sum_{0}^{\infty} x^{n+1} E\left[\exp \left\{i \gamma\left(S_{n}+X_{n+1}\right)\right\} ; M_{n}=0\right] \\
& -\sum_{0}^{\infty} x^{n+1} E\left[\exp \left\{i \gamma\left(S_{n}+X_{n+1}\right)\right\} ; M_{n+1}=0\right] \\
= & {[x \phi(\gamma)-1] \sum_{0}^{\infty} x^{n} E\left[\exp \left\{i \gamma S_{n}\right\} ; M_{n}=0\right]+1, }
\end{aligned}
$$

where

$$
\phi(\gamma)=E\left[e^{i \gamma X}\right]
$$


One can factor $[1-x \phi(\gamma)]^{-1}$ in the following way:

$$
\begin{aligned}
{[1-x \phi(\gamma)]^{-1}=} & \exp \left\{\sum_{1}^{\infty} \frac{x^{k}}{k} E\left[\exp \left\{i \gamma S_{k}\right\} ; S_{k} \geqq 0\right]\right\} \\
& \cdot \exp \left\{\sum_{1}^{\infty} \frac{x^{k}}{k} E\left[\exp \left\{i \gamma S_{k}\right\} ; S_{k}<0\right]\right\} \\
= & \psi_{1}(\gamma ; x) \psi_{2}(\gamma ; x),
\end{aligned}
$$

where $\psi_{1}$ is bounded and regular in the half-plane $\operatorname{Im} \gamma>0$ and $\psi_{2}$ in $\operatorname{Im} \gamma<0$. It follows that

$$
E\left[x^{N} e^{i \gamma Z}-1\right] \psi_{1}(\gamma ; x)=-\sum_{0}^{\infty} x^{n} E\left[e^{i \gamma S_{n}} ; M_{n}=0\right]\left[\psi_{2}(\gamma ; x)\right]^{-1} .
$$

It is easy to check that the left hand side of (3.1) is regular and bounded in $\operatorname{Im} \gamma>0$, the right hand side in $\operatorname{Im} \gamma<0$. Also the boundary values for $\operatorname{Im} \gamma=0$ are assumed continuously by both functions. Therefore they represent together an analytic function, which by Liouville's theorem must be constant. To determine this constant we let $i \gamma=\lambda, \lambda \rightarrow \infty$. The right hand side of equation (3.1) then converges to the first sum in

$$
-\sum_{0}^{\infty} x^{n} \operatorname{Pr}\left[S_{n}=0, M_{n}=0\right]=-\exp \left\{\sum_{1}^{\infty} \frac{x^{k}}{k} \operatorname{Pr}\left\{S_{k}=0\right]\right\} .
$$

The identity of the first and second sums is a special case of Theorem 6.1 in [9]. Substitution into (3.1) completes the proof. We shall also need the following two results:

Corollary 3.2. For $\lambda \geqq 0, E\left(X_{i}\right) \geqq 0$,

$$
E\left[e^{-\lambda Z]}=1-\exp \left\{-\sum_{1}^{\infty} \frac{1}{k} E\left[e^{-\lambda S_{k}} ; S_{k}>0\right]\right\} .\right.
$$

First one can let $x \rightarrow 1$ in Theorem 3.1 since $E\left[x^{N} e^{-\lambda Z}\right]$ is a power series with non-negative coefficients in $x$. The statement then remains correct when $\lambda=0$, since the series

$$
\sum_{1}^{\infty} \frac{1}{k} \operatorname{Pr}\left[S_{k}>0\right]
$$

was shown in [9] to diverge when $E\left(X_{i}\right) \geqq 0$.

Corollary 3.3. The series $\sum_{1}^{\infty}(1 / k) \operatorname{Pr}\left[S_{k}=0\right]$ converges.

This is true for arbitrary random variables $X_{i}$, and can be easily deduced from equation (3.2), which is also quite general. Let $u_{k}=\operatorname{Pr}\left[S_{k}=0, M_{k}=0\right]$. Then one can show that 


$$
\sum_{0}^{\infty} u_{k} x^{k}=[1-F(x)]^{-1}
$$

where

$$
\begin{gathered}
F(x)=\sum_{1}^{\infty} f_{k} x^{k} \\
f_{k}=\operatorname{Pr}\left[S_{1}<0, \cdots, S_{k-1}<0, S_{k}=0\right],
\end{gathered}
$$

and it is obvious that

$$
F(1)=\sum_{1}^{\infty} f_{k}<1
$$

Next we shall find $E(Z)$, which is a rather subtle task.

Theorem 3.4. If $E\left(X_{i}\right)=\mu>0$, then

$$
E(Z)=\mu E(N)=\mu \exp \left\{\sum_{1}^{\infty} \frac{1}{k} \operatorname{Pr}\left[S_{k} \leqq 0\right]\right\} .
$$

If $E\left(X_{i}\right)=0$ and $0<\sigma^{2}\left(X_{i}\right)=\sigma^{2}$, then

$$
E(Z)=\frac{\sigma}{2^{1 / 2}} \cdot c,
$$

where

$$
0<c=\exp \left\{\sum_{1}^{\infty} \frac{1}{k}\left[\frac{1}{2}-\operatorname{Pr}\left(S_{k}>0\right)\right]\right\}<\infty .
$$

Proof. The first part, when $\mu>0$, is a consequence of Wald's fundamental theorem of sequential analysis. We refer to Doob's treatment of this theorem in [5], from which the intuitively plausible result that $E(Z)=\mu E(N)$ follows immediately. Then, from Theorem 3.1 it can be deduced without difficulty that

$$
E(N)=\exp \left\{\sum_{1}^{\infty} \frac{1}{k} \operatorname{Pr}\left(S_{k} \leqq 0\right)\right\}<\infty .
$$

(It was shown in [9] that the series in the exponent converges when $\mu>0$.)

The case when $\mu=0$ cannot be handled by this method. Instead we use a different method, which would also have given the result of the first part. We write 


$$
\begin{aligned}
E(Z) & =\sum_{0}^{\infty} E\left[S_{n+1} ; M_{n}=0 ; M_{n+1}>0\right] \\
& =\lim _{n \rightarrow \infty} \sum_{0}^{n} E\left[S_{k+1} ; M_{k}=0 ; M_{k+1}>0\right] \\
& =\lim _{n \rightarrow \infty} \sum_{0}^{n}\left\{E\left[S_{k+1} ; M_{k}=0\right]-E\left[S_{k+1} ; M_{k+1}=0\right]\right\} \\
& =\lim _{n \rightarrow \infty} \sum_{0}^{n}\left\{E\left[S_{k} ; M_{k}=0\right]-E\left[S_{k+1} ; M_{k+1}=0\right]\right\} \\
& =-\lim _{n \rightarrow \infty} E\left[S_{n} ; M_{n}=0\right] .
\end{aligned}
$$

Let $A_{n}=E\left[S_{n} ; M_{n}=0\right]$, and $A(t)=\sum_{0}^{\infty} A_{n} t^{n}$.

Using Theorem 6.1 of [9] one obtains

$$
\sum_{0}^{\infty} t^{n} E\left[e^{\lambda S_{n}} ; M_{n}=0\right]=\exp \left\{\sum_{1}^{\infty} \frac{t^{k}}{k} E\left[e^{\lambda S_{k}} ; S_{k} \leqq 0\right]\right\} \text {. }
$$

Differentiating with respect to $\lambda$, and setting $\lambda=0$ one gets

$$
\begin{aligned}
A(t)= & \sum_{0}^{\infty} t^{n} E\left[S_{n} ; M_{n}=0\right]=\sum_{1}^{\infty} \frac{t^{k}}{k} E\left[S_{k} ; S_{k} \leqq 0\right] \\
& \cdot \exp \left\{\sum_{1}^{\infty} \frac{t^{k}}{k} \operatorname{Pr}\left[S_{k} \leqq 0\right]\right\} .
\end{aligned}
$$

As $E(Z)=-\lim _{n \rightarrow \infty} A_{n}$, and as it follows from (3.3) that the sequence $-A_{n}$ is monotone and nondecreasing, we have, by a theorem of Hardy and Littlewood

$$
E(Z)=-\lim _{t \rightarrow 1}(1-t) A(t)
$$

It only remains to show that this limit is $\sigma c / 2^{1 / 2}$. Using equation (3.4) one gets

(3.5) $E(Z)=-\lim _{t \rightarrow 1}(1-t)^{1 / 2} \sum_{1}^{\infty} \frac{t^{k}}{k} E\left[S_{k} ; S_{k} \leqq 0\right] \cdot \exp \left\{\sum_{1}^{\infty} \frac{t^{k}}{k}\left(\frac{1}{2}-a_{k}\right)\right\}$

where $a_{k}=\operatorname{Pr}\left[S_{k}>0\right]$.

But the central limit theorem states that

$$
\lim _{k \rightarrow \infty} E\left[\frac{S_{k}}{k^{1 / 2}} ; S_{k} \leqq 0\right]=-\left(\frac{2}{\pi}\right)^{1 / 2} \sigma .
$$

A simple argument from summability theory then shows that 


$$
\lim _{t \rightarrow 1}-(1-t)^{1 / 2} \sum_{1}^{\infty} \frac{t^{k}}{k} E\left[S_{k} ; S_{k} \leqq 0\right]=\frac{\sigma}{2^{1 / 2}} .
$$

Now it only remains to show that

$$
0<\lim _{t \rightarrow 1} \exp \left\{\sum_{1}^{\infty} \frac{t^{k}}{k}\left(\frac{1}{2}-a_{k}\right)\right\}=\exp \left\{\sum_{1}^{\infty} \frac{1}{k}\left(\frac{1}{2}-a_{k}\right)\right\}<\infty,
$$

and this requires some care. We know that

$$
\lim _{t \rightarrow 1} \exp \left\{\sum_{1}^{\infty} \frac{t^{k}}{k}\left(\frac{1}{2}-a_{k}\right)\right\}
$$

exists and is positive. If it is finite, the proof is finished, because then, by Tauber's original theorem,

$$
\sum_{1}^{\infty} \frac{1}{k}\left(\frac{1}{2}-a_{k}\right)=\lim _{t \rightarrow 1} \sum_{1}^{\infty} \frac{t^{k}}{k}\left(\frac{1}{2}-a_{k}\right) .
$$

But suppose that this limit is $+\infty$, i.e. that

$$
\lim _{t \rightarrow 1} \sum_{1}^{\infty} \frac{t^{k}}{k}\left[\frac{1}{2}-\operatorname{Pr}\left(S_{k}>0\right)\right]=+\infty .
$$

Using Corollary 3.3,

$$
0<\lim _{t \rightarrow 1} \sum_{1}^{\infty} \frac{t^{k}}{k} \operatorname{Pr}\left(S_{k}=0\right)<\infty .
$$

Combining (3.6) and (3.7) we have

$$
\lim _{t \rightarrow 1} \sum_{1}^{\infty} \frac{t^{k}}{k}\left[\frac{1}{2}-\operatorname{Pr}\left(S_{k}<0\right)\right]=-\infty
$$

Retracing the entire proof applied to the random variable $Z^{*}$ instead of $Z$ where $Z^{*}$ is the first negative instead of the first positive partial sum, this would imply that $E\left(Z^{*}\right)=0$, which is impossible. The proof is therefore finished.

We will need one more result which has some corollaries of independent interest.

Theorem 3.5. Let $E\left(X_{i}\right)=0,0<\sigma^{2}\left(X_{i}\right)=\sigma^{2}<\infty$,

$$
\begin{aligned}
& p_{0}=q_{0}=1, \quad p_{n}=\operatorname{Pr}\left[S_{1}>0, \cdots, S_{n}>0\right], \quad q_{n}=\operatorname{Pr}\left[S_{1} \leqq 0, \cdots, S_{n} \leqq 0\right] \\
& a_{n}=\operatorname{Pr}\left[S_{n}>0\right] \text { for } n \geqq 1, \quad c=\exp \left\{\sum_{1}^{\infty} \frac{1}{k}\left(\frac{1}{2}-a_{k}\right)\right\} .
\end{aligned}
$$

Then 


$$
\lim _{n \rightarrow \infty}(n \pi)^{1 / 2} p_{n}=\frac{1}{c}
$$

and

$$
\lim _{n \rightarrow \infty}(n \pi)^{1 / 2} q_{n}=c .
$$

Proof. It is known [9] that

$$
\sum_{0}^{\infty} p_{n} t^{n}=\exp \left\{\sum_{1}^{\infty} \frac{a_{k}}{k} t^{k}\right\}
$$

In the proof of the last theorem it was shown that the series

$$
\sum_{1}^{\infty} \frac{1}{k}\left(\frac{1}{2}-a_{k}\right)
$$

converges to $\log c$ (conditionally; it probably need not converge absolutely). Therefore

$$
\lim _{t \rightarrow 1}(1-t)^{1 / 2} \sum_{0}^{\infty} p_{n} t^{n}=\frac{1}{c}
$$

This together with the fact that $p_{n} \geqq p_{n+1}$ for $n=0,1, \cdots$ suffices to conclude (see $[10$, p. 339$])$ that

$$
\lim _{n \rightarrow \infty}(n \pi)^{1 / 2} p_{n}=\frac{1}{c}
$$

The proof of the second statement follows in the same way from

$$
\sum_{0}^{\infty} q_{n} t^{n}=\exp \left\{\sum_{1}^{\infty} \frac{t^{k}}{k}\left(1-a_{k}\right)\right\} .
$$

The theorem has the following interesting by-products, which however will not be needed in the sequel.

Corollary 3.6. If $E\left(X_{i}\right)=0,0<\sigma^{2}\left(X_{i}\right)=\sigma^{2}<\infty$, then

(a)

$$
\lim _{n \rightarrow \infty} E\left[\frac{S_{n}}{\left(n \sigma^{2}\right)^{1 / 2}} \mid S_{1} \geqq 0, S_{2} \geqq 0, \cdots, S_{n} \geqq 0\right]=\left(\frac{\pi}{2}\right)^{1 / 2},
$$

(b)

$$
\lim _{n \rightarrow \infty} E\left[S_{n} \mid \text { at least one of } S_{1}, S_{2}, \cdots, S_{n} \text { is }>0\right]=\frac{\sigma}{2^{1 / 2}} c .
$$

The proof is straight-forward and therefore omitted. The first part gains 
in interest by comparison to the (trivial) fact that $\left({ }^{2}\right)$

$$
\lim _{n \rightarrow \infty} E\left[\frac{S_{n}}{\left(n \sigma^{2}\right)^{1 / 2}} \mid S_{n} \geqq 0\right]=\left(\frac{2}{\pi}\right)^{1 / 2} .
$$

Finally we mention an apparently new form of the celebrated arc-sine law for arbitrary random variables with mean zero and finite variance.

Corollary 3.7. Let $N_{n}$ be the number of positive partial sums among $S_{1}, S_{2}, \cdots, S_{n}$, in the case when $E\left(X_{i}\right)=0,0<\sigma^{2}\left(X_{i}\right)=\sigma^{2}<\infty$. Then

$$
[k(n-k)]^{1 / 2} \operatorname{Pr}\left[N_{n}=k\right]=\frac{1}{\pi}+o(1),
$$

where $o(1) \rightarrow 0$ as $\min (k, n-k) \rightarrow \infty$.

The proof follows directly from Theorem 3.5 and from Sparre Andersen's theorem, [1], which states that

$$
\operatorname{Pr}\left[N_{n}=k\right]=p_{k} q_{n-k} .
$$

4. Now we are in a position to find an analytical representation for the solution $F(x)$ of equation (1.1).

In accordance with the notation in $\$ 2$, we introduce the distribution functions

$$
\begin{aligned}
F_{n}(x) & =\operatorname{Pr}\left[T_{n} \leqq x \mid T_{0}=0\right]=\operatorname{Pr}\left[M_{n} \leqq x\right], \\
{ }_{0} F_{n}(x) & =\operatorname{Pr}\left[T_{n} \leqq x ; T_{i} \neq 0 \text { for } i=1, \cdots, n-1 \mid T_{0}=0\right] \\
& =\operatorname{Pr}\left[S_{n} \leqq x, S_{i}>0 \text { for } i=1, \cdots, n-1\right] \text { for } n \geqq 1, x \geqq 0 . \\
{ }_{0} F_{0}(x) & =1 \\
& \text { for } x \geqq 0,0 \text { for } x<0 .
\end{aligned}
$$

Using (4.2) a simple renewal argument shows that

$$
F_{n}(x)=\sum_{k=0}^{n-1} F_{k}(0){ }_{0} F_{n-k}(x)=\sum_{k=1}^{n} F_{n-k}(0)_{0} F_{k}(x), \quad \text { for } n \geqq 1, x \geqq 0,
$$

or

$$
F_{n}(x)=\sum_{k=0}^{n} F_{n-k}(0) \cdot{ }_{0} F_{k}(x)-F_{n}(0)+\delta_{n 0}, \quad n \geqq 0, x \geqq 0 .
$$

Now we form the generating function $(0 \leqq t<1)$

$$
\sum_{1}^{\infty}{ }_{0} F_{n}(x) t^{n}=\frac{\sum_{0}^{\infty} F_{n}(x) t^{n}}{\sum_{0}^{\infty} F_{n}(0) t^{n}}-\frac{1}{\sum_{0}^{\infty} F_{n}(0) t^{n}}, \quad x \geqq 0 .
$$

${ }^{(2)}$ Added in proof. This phenomenon is explained by the following generalization of part (a) of Corollary 3.6. If $E\left(X_{i}\right)=0,0<\sigma^{2}\left(x_{i}\right)=\sigma^{2}<\infty$, then $\lim _{n \rightarrow \infty} \operatorname{Pr}\left[S_{n} /\left(n \sigma^{2}\right)^{1 / 2} \leqq x \mid S_{1}\right.$ $\left.\geq 0, \cdots, S_{n} \geqq 0\right]=1-e^{-x^{2} / 2}, 0 \leqq x<\infty$. 
It is clear that $F_{n}(0)=q_{n}$ so that by Theorem 3.5

$$
\lim _{n \rightarrow \infty}(n \pi)^{1 / 2} F_{n}(0)=\lim _{t \rightarrow 1}(1-t)^{1 / 2} \sum_{0}^{\infty} F_{n}(0) t^{n}=c .
$$

Since ${ }_{0} F_{n}(x) \geqq 0$, equation (4.3) implies that

$$
\lim _{t \rightarrow 1} \sum_{1}^{\infty}{ }_{0} F_{n}(x) t^{n}=\sum_{1}^{\infty}{ }_{0} F_{n}(x)=\lim _{t \rightarrow 1} \frac{1}{c}(1-t)^{1 / 2} \sum_{0}^{\infty} F_{n}(x) t^{n} .
$$

To show that this limit is finite one can take Laplace transforms on equation (4.3). Then

$$
\begin{aligned}
\sum_{1}^{\infty} t^{n} \int_{0-}^{\infty} e^{-\lambda x} d_{0} F_{n}(x) & =\frac{\exp \left\{\sum_{1}^{\infty} \frac{t^{k}}{k} E\left[e^{-\lambda S_{k}} ; S_{k}>0\right]+\operatorname{Pr}\left[S_{k} \leqq 0\right]\right\}}{\exp \left\{\sum_{1}^{\infty} \frac{1}{k} \operatorname{Pr}\left(S_{k} \leqq 0\right) t^{k}\right\}} \\
= & \exp \left\{\sum_{1}^{\infty} \frac{t^{k}}{k} E\left[e^{-\lambda S_{k}} ; S_{k}>0\right]\right\}=\frac{1}{1-E\left[t^{N} e^{-\lambda Z}\right]} .
\end{aligned}
$$

Here we have used Theorem 3.1 of [9] and Theorem 3.1 of $\$ 3$.

It follows that

$$
\lim _{t \rightarrow 1} \sum_{1}^{\infty} t^{n} \int_{0-}^{\infty} e^{-\lambda x} d_{0} F_{n}(x)=\frac{1}{1-E\left[e^{-\lambda Z}\right]}, \quad \lambda>0 .
$$

By the continuity theorem for Laplace transforms proved in Lemma 7, [10], it follows that

$$
\text { (4.7) } \lim _{t \rightarrow 1} \sum_{1}^{\infty}{ }_{0} F_{n}(x) t^{n}=\sum_{1}^{\infty}{ }_{0} F_{n}(x)=1+\sum_{1}^{\infty} \operatorname{Pr}\left[Z_{1}+\cdots+Z_{n} \leqq x\right]<\infty \text {, }
$$

where $Z_{i}$ are identically distributed random variables with the same distribution as $Z\left(^{(3)}\right.$. Also, using the same argument as in the proof of Theorem 3.5 , the monotonicity of the sequence $F_{n}(x)$ implies that

$$
\sum_{1}^{\infty}{ }_{0} F_{n}(x)=\lim _{n \rightarrow \infty} \frac{(n \pi)^{1 / 2}}{c} F_{n}(x) .
$$

The main result of this section can now be stated. If we define

$$
G(x)=\lim _{n \rightarrow \infty}(n \pi)^{1 / 2} F_{n}(x)=c \cdot \sum_{1}^{\infty}{ }_{0} F_{n}(x),
$$

(3) The random variables $Z_{1}+Z_{2}+\cdots+Z_{n}$ used here and in the sequel were called the ladder random variables of the sequence $S_{n}$ by D. Blackwell [ 3 ], who used them to extend the renewal theorem to arbitrary random variables with positive mean. 
then one can assert

THEOREM 4.1. $G(x)$ is a nondecreasing right-continuous solution of equation (1.1). It is absolutely continuous if $K(x)$ is.

The simple proof is taken directly from [10]. The definition of ${ }_{0} F_{n}(x)$ implies that

$$
\int_{0-}^{\infty} K(x-y) d_{0} F_{n}(x)=K(x)_{0} F_{n}(0)+{ }_{0} F_{n+1}(x), \quad n \geqq 1 .
$$

Summing on $n$ yields, by equation (4.9)

$$
\sum_{1}^{\infty} \int_{0-}^{\infty} K(x-y) d_{0} F_{n}(y)=\frac{1}{c} K(x) G(0)+\frac{1}{c} G(x)-{ }_{0} F_{1}(x) .
$$

Equation (4.2) shows that

$$
\sum_{1}^{\infty}{ }_{0} F_{n}(0)=\frac{1}{c} G(0)=1,
$$

so that

$$
\begin{aligned}
\sum_{1}^{\infty} \int_{0-}^{\infty} K(x-y) d_{0} F_{n}(y) & =K(x)+\frac{1}{c} G(x)-{ }_{0} F_{\mathrm{i}}(x) \\
& =\frac{1}{c} G(x) .
\end{aligned}
$$

Finally the integrands above are non-negative which justifies interchanging summation and integration to conclude that

$$
\int_{0-}^{\infty} K(x-y) d G(y)=G(x) .
$$

The remaining statements of the theorem follow from (4.2) and (4.9).

5. In this section we conclude the proof of Theorem 1 which was stated in the introduction. The distributions $F_{n}(x)$ defined in the introduction can easily be shown to be the same as those denoted, carelessly, also by $F_{n}(x)$ in equation (4.1). In fact, one can easily do more, namely verify that when the $X_{i}$ do not have a density, and if

$$
\begin{aligned}
F_{0}(x) & =1, & x \geqq 0, \\
F_{n+1}(x) & =\int_{0-}^{\infty} K(x-y) d F_{n}(y), & x \geqq 0, n \geqq 0,
\end{aligned}
$$

then

$$
F_{n}(x)=\operatorname{Pr}\left[M_{n} \leqq x\right] .
$$

In view of this and Theorems 1.1, 2.1, and 4.1, we have now established 
all of Theorem 1 with the exception of equation (0.3). In so doing we have of course obtained analogous results for arbitrary random variables. Only at this point do we have to make a distinction, namely between

CASE (a) the random variables $X_{i}$ have a nonlattice distribution, with $E\left(X_{i}\right)=0$ and $0<\sigma^{2}\left(X_{i}\right)=\sigma^{2}<\infty$ and

CASE (b) the random variables $X_{i}$ have a lattice distribution and it is assumed, without loss of generality, that the smallest additive subgroup of the real line containing the points of increase of the distribution $K(x)$ is the group of all integers. In addition we assume of course that $E\left(X_{i}\right)=0$ and $0<\sigma^{2}\left(X_{i}\right)=\sigma^{2}<\infty$.

This distinction depends on the need to apply different forms of the renewal theorem. Corresponding to Case (a) we shall prove

TheOREM 5.1a. In Case (a) the solution $G(x)$ of equation (1.1) as defined in Theorem 4.1 has the asymptotic behavior

$$
\lim _{x \rightarrow \infty}[G(x+h)-G(x)]=h \frac{2^{1 / 2}}{\sigma} .
$$

In Case (b) it is clear from the definition of $G(x)$ in equation (4.9) that $G(x)$ is a step function with positive jumps at the points $x=n=0,1,2, \ldots$. Letting

$$
\begin{aligned}
G_{n} & =G\left(n^{+}\right), \\
K(n) & =\sum_{k=-\infty}^{n} C_{k},
\end{aligned}
$$

where $C_{k}=\operatorname{Pr}\left[X_{i}=k\right]$, equation (1.1) in this case reduces to

$$
G_{n}=\sum_{k=0}^{\infty} C_{n-k} G_{k}
$$

and it is the monotone solution of (5.2) which according to Theorem 4.1 is unique, up to a constant. Its limiting behavior will be given by

Theorem 5.1 (b). In Case (b) the solution $G_{n}$ of equation (5.2) as defined in Theorem 4.1 has the asymptotic behavior

$$
\lim _{n \rightarrow \infty}\left[G_{n+1}-G_{n}\right]=\frac{2^{1 / 2}}{\sigma} .
$$

Proof. In Case (a) as well as (b) equations (4.6) and (4.9) together imply that equation (0.5) holds, i.e. that

$$
\int_{0-}^{\infty} e^{-\lambda x} d G(x)=\frac{c}{1-E\left[e^{-\lambda Z}\right]} .
$$

This says that, for $x \geqq 0$ 


$$
\frac{1}{c} G(x)=1+\sum_{k=1}^{\infty} \operatorname{Pr}\left[Z_{1}+\cdots+Z_{k} \leqq x\right]
$$

where the $Z_{i}$ are identically distributed independent random variables with the same distribution as $Z$. In Case (a) we use the renewal theorem which was first proved by Blackwell [3] in sufficient generality to conclude that

$$
\lim _{x \rightarrow \infty} \frac{1}{c}[G(x+h)-G(x)]=\frac{h}{E(Z)} .
$$

This concludes the proof, since Theorem 3.4 has shown that $E(Z)=\sigma / 2^{1 / 2} c$.

In the lattice case the proof is the same, using the renewal theorem in the form due to Feller [6].

Finally, we specialize to those cases when the Laplace transform of $G(x)$ has an interesting analytical representation, namely when the random variables $X_{i}$ are symmetric. We shall consider the two cases:

(A) $K(x)$ is continuous, with $K(x)=1-K(-x)$,

(B) $K(x)=\sum_{k=-\infty}^{[x]} C_{k}$ with $C_{k}=C_{-k}$, and g.c.d. $\left[k \mid C_{k}>0\right]=1$, and of course the mean is 0 and the variance finite, as before. Then we can state Tauberian theorems, which, on the surface, seem to have nothing to do with the Wiener-Hopf equation.

Theorem 5.2 A. If $G(x)$ has the Laplace transform

$$
1+\int_{0+}^{\infty} e^{-\lambda x} d G(x)=\exp \left\{-\frac{1}{\pi} \int_{0}^{\infty} \frac{\lambda}{\lambda^{2}+u^{2}} \log [1-\phi(u)] d u\right\},
$$

where

$$
\phi(u)=\int_{-\infty}^{\infty} e^{i \lambda x} d K(x), \text { with } K(x) \text { of type } \mathrm{A},
$$

then $\lim _{x \rightarrow \infty}[G(x+h)-G(x)]=2^{1 / 2} / \sigma \cdot h$.

THEOREM 5.2B. If $u_{n}$ are the coefficients in

$$
\sum_{0}^{\infty} u_{k} t^{k}=\exp \left\{-\frac{1}{2 \pi} \int_{0}^{\pi} \frac{1-t^{2}}{1+t^{2}-2 t \cos \theta} \log [1-\phi(\theta)] d \theta\right\},
$$

where $\phi(\theta)=\sum_{-\infty}^{\infty} C_{k} e^{i k \theta}$, with $C_{k}$ of type $\mathrm{B}$, then $\lim _{n \rightarrow \infty} u_{n}=2^{1 / 2} / \sigma$.

The proof of part $\mathrm{A}$ is an immediate consequence of a trivial extension of equation (0.4) and of Theorem 5.1a. Therefore we discuss only part B. We shall make the identification

$$
u_{0}=G_{0}, \quad u_{n}=G_{n}-G_{n-1}, \quad n \geqq 1,
$$


where $G_{n}=G\left(n^{+}\right)$, and $G(x)$ is the solution of (5.2) in Theorem 5.1b. This time we use equation (4.5) which gives

$$
\begin{aligned}
\sum_{0}^{\infty} u_{n} e^{-\lambda n} & =\int_{0-}^{\infty} e^{-\lambda x} d G(x)=c \sum_{1}^{\infty} \int_{0-}^{\infty} e^{-\lambda x} d_{0} F_{n}(x) \\
& =c \exp \left\{\sum_{1}^{\infty} \frac{1}{k} E\left[e^{-\lambda S_{k}} ; S_{k}>0\right]\right\} .
\end{aligned}
$$

But, since $\operatorname{Pr}\left[X_{i}=k\right]=\operatorname{Pr}\left[X_{i}=-k\right]$, we have

$$
\frac{1}{2}-\operatorname{Pr}\left[S_{k}>0\right]+E\left[e^{-\lambda S_{k}} ; S_{k}>0\right]=\frac{1}{2} E\left[e^{-\lambda\left|S_{k}\right|}\right] \text {. }
$$

Therefore

$$
\sum_{0}^{\infty} u_{n} t^{n}=\exp \left\{\frac{1}{2} \sum_{1}^{\infty} \frac{1}{k} E\left[e^{-\lambda\left|S_{k}\right|}\right]\right\} .
$$

Hence Theorem 5.2B will follow from Theorem 5.1b, provided that it turns out that

$$
\sum_{1}^{\infty} \frac{1}{k} E\left[t^{\left|S_{k}\right|}\right]=-\frac{1}{\pi} \int_{0}^{\pi} \frac{1-t^{2}}{1+t^{2}-2 t \cos \theta} \log [1-\phi(\theta)] d \theta .
$$

One shows this as follows. For $0 \leqq t<1,0 \leqq x<1$

$$
\begin{aligned}
\sum_{1}^{\infty} \frac{x^{k}}{k} E\left[t^{\left|S_{k}\right|}\right] & =\sum_{k=1}^{\infty} \frac{x^{k}}{k} \sum_{\nu=-\infty}^{\infty} t^{|\nu|} \frac{1}{2 \pi} \int_{-\pi}^{\pi} e^{-i \theta \nu} \phi^{k}(\theta) d \theta \\
& =\frac{1}{2 \pi} \sum_{k=1}^{\infty} \frac{x^{k}}{k} \int_{-\pi}^{\pi} \frac{1-t^{2}}{1+t^{2}-2 t \cos \theta} \phi^{k}(\theta) d \theta \\
& =-\frac{1}{\pi} \int_{0}^{\pi} \frac{1-t^{2}}{1+t^{2}-2 t \cos \theta} \log [1-x \phi(\theta)] d \theta .
\end{aligned}
$$

To let $x \rightarrow 1$ requires some justification. First one shows that

$$
\lim _{x \rightarrow 1} \sum_{1}^{\infty} \frac{x^{k}}{k} E\left[t\left|S_{k}\right|\right]=\sum_{1}^{\infty} \frac{1}{k} E\left[t\left|S_{k}\right|\right]<\infty,
$$

for every $0 \leqq t<1$. One can show this easily if one uses the strong result in [4] that there exists an absolute constant $A$, independent of $k$, such that

$$
\operatorname{Pr}\left[S_{n}=k\right] \leqq A / n^{1 / 2} \text { for all } k, n \text {. }
$$

Hence we know that

$$
-\lim _{x \rightarrow 1} \frac{1}{\pi} \int_{0}^{\pi} \frac{1-t^{2}}{1+t^{2}-2 t \cos \theta} \log [1-x \phi(\theta)] d \theta=\sum_{1}^{\infty} \frac{1}{k}\left\{E t^{\left|S_{k}\right|}\right\}<\infty .
$$


Now we decompose the Poisson integral into two integrals over the set $A=[\theta \mid 0 \leqq \theta \leqq \pi ; \phi(\theta)<0]$ and its complement $B$. Clearly the limit operation $(x \rightarrow 1)$ and integration over $A$ can be interchanged, since the logarithm is bounded there. Over $B$ limit and integration can be interchanged by the dominated convergence theorem, since $\log [1-\phi(\theta)] \in L_{1}(0,2 \pi)$. This follows from the fact that $\phi(\theta)=1-\sigma^{2} \theta^{2} / 2+o\left(\theta^{2}\right)$ as $\theta \rightarrow 0$. That completes the proof of (5.4) and of this theorem.

6. By reinterpreting the asymptotic result of Theorem 5.1 it will now be shown that we can extend the basic theorem of the theory of recurrent events, as explained in the introduction. It seems natural to consider the asymptotic behavior of the sum

$$
S(x, h)=\sum_{n=0}^{\infty} \operatorname{Pr}\left[x<S_{n} \leqq x+h ; M_{n-1}<S_{n}\right],
$$

for the following reason. When the random variables $X_{i}$ are positive, $S(x, h)$ reduces to

$$
S(x ; h)=\sum_{n=0}^{\infty} \operatorname{Pr}\left[x<S_{n} \leqq x+h\right] .
$$

When, on the other hand, the $X_{i}$ are arbitrary nonlattice random variables with finite mean $\mu \geqq 0,(6.1)$ is a natural generalization or (6.2). Only those values of $S_{n}$ are counted, we shall call them the renewal points of the process, which exceed all the previous values. Our results will depend on the obvious fact that the distances between two successive renewal points are mutually independent. We shall call them $Z_{1}, Z_{2}, \ldots$ as it is obvious that each of them has the same distribution as the random variable $Z$ of $\S 3$, the first positive partial sum. Summarizing we have

$$
\begin{aligned}
S(x, h) & =\sum_{n=1}^{\infty} \operatorname{Pr}\left[x<S_{n} \leqq x+h ; M_{n-1}<S_{n}\right] \\
& =\sum_{n=1}^{\infty} \operatorname{Pr}\left[x<Z_{1}+\cdots+Z_{n} \leqq x+h\right] .
\end{aligned}
$$

The renewal theorem for positive random variables gives therefore

$$
\lim _{x \rightarrow \infty} S(x, h)=h / E(Z),
$$

and Theorem 3.4 then gives equations $(0.9)$ and $(0.10)$.

Equation (0.8) is a renewal theorem of a different type. For random variables with positive mean, which are not necessarily positive, the sum

$$
\sum_{n=0}^{\infty} \operatorname{Pr}\left[x<M_{n} \leqq x+h\right]
$$


is a generalization of the sum in (6.2) which is different from and always greater than or equal to $S(x, h)$. Intuition suggests, and it is easy to show, by going back to Theorem 3.1 that

$$
\sum_{0}^{\infty} E\left[e^{-\lambda M_{n}}\right]=\frac{E[N]}{1-E\left[e^{-\lambda Z}\right]} .
$$

The renewal theorem for positive random variables together with Theorem 3.4 yields

$$
\lim _{x \rightarrow \infty} \sum_{0}^{\infty} \operatorname{Pr}\left[x<M_{n} \leqq x+h\right]=\frac{h E(N)}{E(Z)}=\frac{h}{\mu} .
$$

This is equation (0.8) and therefore we have proved

THEOREM 6.1. For arbitrary nonlattice random variables with finite expected value $\mu$, equations (0.8) and (0.9) hold if $\mu>0$, and equation (0.10) holds if $\mu=0$ and if in addition the variance $\sigma^{2}$ is finite.

\section{REFERENCES}

1. E. Sparre Andersen, On the fluctuations of sums of random variables II, Math. Scand. vol. 2 (1954) pp. 195-223.

2. G. Baxter, An operator identity, Pacific J. Math. vol. 8 (1958) pp. 649-663.

3. D. Blackwell, Extension of a renewal theorem, Pacific J. Math. vol. 3 (1953) pp. 315-332.

4. K. L. Chung and P. Erdös, Probability limit theorems assuming only the first moment I, Memoirs Amer. Math. Soc., no. 6, 1951.

5. J. L. Doob, Stochastic processes, New York, 1953. 1950.

6. W. Feller, An introduction to probability theory and its applications, vol. 1, New York,

7. T. E. Harris, The existence of stationary measures for certain Markov processes, Proceedings of the Third Berkeley Symposium, vol. 2, 1956, pp. 113-123.

8. F. Pollaczek, Fonctions characteristiques de certaines repartitions definies an moyen de la notion d'ordre, C. R. Acad. Sci. Paris vol. 234 (1952) pp. 2334-2336.

9. F. Spitzer, A combinatorial lemma and its application to probability theory, Trans. Amer. Math. Soc. vol. 82 (1956) pp. 323-339.

10. - The Wiener-Hopf equation whose kernel is a probability density, Duke Math. J. vol. 24 (1957) pp. 327-344.

11. F. Spitzer and C. Stone, Some applications of Toeplitz matrices to probability theory (to appear in Illinois J. Math.).

UNIVERSity OF MiNNESOTA, MinneAPolis, MinNESOTA 